E. Zur Bestimmungdes Molekulargewichts

Unabhängig von jeder Theorie kann man offenbar den Dampfdruck bis zu ziemlich hohen Konzentrationen durch die Formel

$$
\ln a_{1}=\ln \frac{P x_{1}^{\prime}}{P x_{1}^{\prime}+x_{2}^{\prime}}-K x_{2}^{\prime 2}
$$

wiedergeben. Für hinreichend verdünnte Lösungen, wie man sie für Molekulargewichtsbestimmungen immer verwendet, kann man den Logarithmus unter Vernachlässigung der höheren Glieder entwickeln; mit der relativen Dampfdruckerniedrigung $b_{1}=1-a_{1}$ erhält man

$$
b_{1} / x_{2}{ }^{\prime}=K x_{2}{ }^{\prime}+1 / P,
$$

also eine lineare Beziehung zwischen $b_{1} / x_{2}{ }^{\prime}$ und $x_{2}{ }^{\prime}$; entsprechendes gilt natürlich für den Zusammenhang zwischen den anderen osmotischen Effekten und der Konzentration. Es ist das die bekannte, von Ostwald herrührende Formel zur Bestimmung des Molekulargewichtes durch Extrapolation. Sie ergibt sich also aus einer Dampfdruckformel, die in dem weiten Bereich zwischen $x=0$ und 70 Grundmolprozenten experimentell erprobt ist; daran ändert sich auch nichts, wenn man für verdünnte Lösungen an Stelle von $x_{2}{ }^{2}$ den Wert $x_{2}{ }^{\prime 2} / x_{1}{ }^{2}$ auf Grund der Entmischungsversuche setzt. Man sollte also nur mit dieser Formel auf unendliche Verdünnung extrapolieren.

\title{
Elektroakustische Messungen an berühmten Barockorgeln Oberschwabens
}

\author{
I. Schalldruckaufnahmen \\ VON WERNER LOTTERMOSER \\ Aus dem Physikalischen Institut der Universität Tübingen \\ (Z. Naturforschg. 3 a, 298-308 [1948]; eingegangen am 16. Januar 1948)
}

\begin{abstract}
Es werden Schalldruckmessungen mitgeteilt, die zeigen, daß die Barockorgeln infolge des geringen Winddrucks relativ schwache Schalldrucke abgeben. Die gemessenen Werte der Plenumklänge erreichen höchstens 1,8 $\mu \mathrm{b}$ im Pedal. Besonders bemerkenswert ist eine äußerst feine Schalldruckdifferenzierung der einzelnen Register, wodurch eine Fülle unterschiedlicher Klangfarben bester Ausgeglichenheit erreicht wird. Die Erzeugung ähnlicher Druckverhältnisse durch moderne Orgeln scheint schwierig zu sein, wird aber in seltenen Fällen doch erzielt, wobei dann die Wirkung ähnlich günstig ist wie bei den Barockorgeln. Es folgt daraus, daß die Nachahmung der gezeigten Schalldruckdiagramme bei modernen Orgeln als erstrebenswertes Ziel anzusehen ist.
\end{abstract}

$\mathrm{D}_{\mathrm{H}}^{\mathrm{i}}$ ie Erforschung der berühmten Orgeln der Barockzeit ist für den Akustiker ganz besonders reizvoll, weil diese Orgeln Klangwirkungen erzeugen, die noch heute, nach rd. 200 Jahren, trotz aller Fortschritte der Technik, als unübertroffen gelten können. Die Frage, warum diese Orgeln so prachtvoll klingen, drängt zur Beantwortung, zumal die bisher auf diesem Gebiet erschienenen Arbeiten ${ }^{1}$ bereits gezeigt haben, daß die Lösung solcher Probleme mit den Mitteln der Elektroakustik möglich ist. Besonders wichtig ist die Anwendung einer vergleichenden Arbeits-

1 E. Thienhaus u. W. Willms, Musik und Kirche 5, 199 [1933]; F. Trendelen bur g, E. Thi en ha s u. E. Franz, Akust. Z. 1, 59 [1936]; 3, 7 [1938]; W. L o t t e r m os er, Akust. Z. 2, 129 [1937]; 3, 63 [1938]; 5, 324 [1940]. weise in der Art, daß die gleichen Meßverfahren bei der Untersuchung guter und schlechter Instrumente benutzt werden. Auf diese Weise kommt man zu sicheren Ergebnissen, die den heutigen Orgelbauer in die Lage versetzen, Instrumente höchster Klanggüte zu erzeugen, die darüber hinaus aber unser Verstắndnis von der physiologischen Wirkung physikalisch definierter Klänge erweitern.

Neben den Einschwingvorgängen und den Klangspektren ${ }^{2}$ ist der Schalldruck eine der wesentlichen Bestimmungsgrößen des Orgelklangs. Beim Niederdrücken einer Taste erzeugt die zugehörige Pfeife bzw. der Pfeifenchor nach einigen Zehntelsekunden des Einschwingens

${ }^{2}$ W. L o tt er m o s e r, Physik. Blätter 3, 103 [1948]. 
einen Klang gleichbleibender Intensität, die von der Pfeifenbauart und der Größe des anblasenden Winddrucks abhängt. Durch Messung des Schalldrucks erhält man Angaben, wie laut die einzelnen Stimmen der Orgel sind, wie sich der Schalldruck innerhalb eines Registers längs der Tonskala verhält und wie er ansteigt, wenn mehrere Register zu Kombinationen vereint werden. Ganz besonders interessiert der Schalldruckverlauf des sogen. Plenums, das sich aus den Prinzipalen, Oktaven und Mixturen ${ }^{3}$ zusammensetzt. Sind mehrere Manuale vorhanden, so ist die Kenntnis ihres Plenums, natürlich auch die des Pedals, wichtig.

Der Schalldruck wird in dem in der Akustik üblichen $\mathrm{Maß}$ von $1 \mu \mathrm{b}=10^{-6}$ Bar angegeben, wobei einem Bar ungefähr der Druck einer Atmosphäre entspricht. Zum Unterschied gegen das subjektive Maß des Phon ${ }^{1}$ hat das $\mu$ bar den Vorteil, das absolute, physikalische $\mathrm{Ma} \bar{\beta}$ darzustellen, so daß die Beziehungen zwischen den voneinander abhängigen Größen: Schalldruck, Pfeifenmensur, Winddruck und Raumakustik besser erfaßbar sind.

Der gemessene Schalldruck ist das Ergebnis einer Überlagerung der harmonisch zueinander liegenden Teiltöne, deren Größe und Zahl bei den Registern sehr verschieden ist. So liefern z. B. die gedeckten Pfeifen meist obertonarme, Zungenpfeifen obertonreiche Klänge. Natürlich gibt die Angabe des Schalldrucks allein keinen Aufschluß über die Teiltonzusammensetzung der Klänge. Die Darstellung der an den Orgeln gemessenen Klangspektren muß einem weiteren Bericht vorbehalten bleiben. Da die akustischen Eigenschaften des Raumes den Orgelklang beeinflussen ${ }^{4}$, ist es zweckmäßig, derartige Messungen immer mitten im Kirchenraum anzustellen, dort,

$3 C$ eines 8 -Registers hat den Grundton $C$ (Normallage)

$C$ eines 16'-Registers hat den Grundton $C_{1}$ (Unteroktave)

$C$ eines 4'-Registers hat den Grundton $c$

(Oberoktave, 2. Teilton)

$C$ eines $2^{\prime}$-Registers hat den Grundton $c^{\prime}$

(Doppeloktave, 4. Teilton)

$C$ eines $2^{2} / 3^{\prime}$-Registers hat den Grundton $g$

(Duodezime, 3. Teilton)

$C$ eines 3'-Registers (alte Bezeichnung) hat den Grundton $g$ (Duodezime, 3. Teilton)

$C$ eines $11^{1} / 2^{\prime}$-Registers hat den Grundton $g^{\prime}$

(6. Teilton)

$C$ eines $1^{3} / 5^{\prime}$-Registers hat den Grundton $e^{\prime}$

(5. Teilton) wo sich der Zuhörer aufhält, denn auf die akustische Wirkung der Orgel an diesen Stellen kommt es an. Wenn beispielsweise in der Kirche große Flächen vorhanden sind, welche den Schall tiefer Frequenzen besonders stark absorbieren, dann kann wohl direkt an der Orgelpfeife ein großer Schalldruck erzeugt und gemessen werden; im Raume aber können die Baßtöne durch die genannte Absorptionswirkung so geschwächt sein, daß die Wirkung der Orgel unbefriedigend ist. Die Kunst des Orgelbauers besteht in nicht geringem Maß darin, Intonation und Mensur der Pfeifen dem Raume so anzupassen, daß am Ohr des Zuhörers die gewünschten Schalldrucke entstehen. Eine kleine Orgel in kleinem Raume soll naturgemäß eine ebenso befriedigende Wirkung haben wie eine große in großem Raume. Deshalb muß natürlich die Schalleistung der Orgel im großen Raum größer sein als die in kleinen Räumen, der Schalldruck am Ohr des Zuhörers soll dagegen, unabhängig von der Raumgröße, Werte besitzen, welche den besten physiologischen Effekt verursachen. Man kann deshalb die Schalldruckkurven von Orgeln verschiedener Größe miteinander vergleichen, und es ist möglich, die Werte, welche an einer Orgel oder besser an mehreren klanglich berühmten Orgeln, gemessen wurden, allgemein als Vorbilder für Neubauten bzw. bei der Korrektur unbefriedigender Klänge heranzuziehen.

\section{Kurze Beschreibung der Orgeln}

Untersucht wurden zwei Orgeln von K. J. Riepp (große Orgel: 49 klingende Stimmen, kleine Orgel: 27 klingende Stimmen), die er in den Jahren 1757-1766 in der Basilika der Benediktiner-Abtei zu Ottobeuren gebaut hat. Da Riepp mehrere Orgeln in Frankreich erstellt und dort wertvolle Anregungen erhalten hat, besitzen seine Werke die besten Eigenschaften deutscher und französischer Orgelbaukunst der damaligen Zeit. Riepps Schüler war J.' N. Holzhay, der die andere vermessene Orgel mit 42 klingenden Stimmen in "der Klosterkirche zu Obermarchtal 1784 baute. Obwohl die Werke der beiden Meister viel ähnliche Züge besitzen, deuten doch etliche, auch physikalisch nachweisbare Unterschiede darauf hin, daß sich das Klangideal der Barockzeit gegen Ende der Epoche etwas wandelte. Davon ab-

${ }^{4} \mathrm{~W}$. L o t t e r m o s e r, Instrumentenbau-Z. 2. 14 [1947]. 
gesehen, stehen aber die drei untersuchten Orgeln auf einer solchen Stufe, daß sie insgesamt unbedenklich als Muster klanglicher Höchstleistung dienen können.

In der Literatur sind sie bereits durch Veröffentlichungen kunsthistorisch-musikalischen Charakters bekannt ${ }^{5}$, und ihre Mensuren sind eingehend gemessen und mitgeteilt worden. Die Riepp-Orgeln befinden sich in fast originalem Zustand. Die Holzhay-Orgel hat infolge der im ersten Weitkrieg angeordneten Zinnabgabe den Verlust einiger wichtiger Stimmen erleiden müssen. Offenbar ist aber der Ersatz durch Zinkpfeifen in einer solchen Weise vorgenommen worden, daß keine Einbuße der klanglichen Schönheit bemerkbar ist. Alle untersuchten Orgeln besitzen Schleifladen, die, wie bekannt, eine wesentliche Voraussetzung hoher Klangqualität bilden. Da sich diese Konstruktion besonders auf die Einschwingvorgänge auswirkt, soll im Rahmen dieser Arbeit nicht näher darauf eingegangen werden. Dagegen ist der Winddruck für den Schalldruck, von großer Bedeutung. Er wurde an den Laden gemessen: bei den Riepp-Orgeln zu $83 \mathrm{~mm}$, bei der Holzhay-Orgel zu $62 \mathrm{~mm}$ Wassersäule. Es ist nicht sicher, ob die Riepp-Orgeln ihren ursprünglichen Winddruck behalten haben, da Veränderungen der Zuführungskanäle stattgefunden haben. Jedenfalls sind die Winddrucke aller 3 Werke gering. Bei vollgriffigem Spiel mit den Hauptregistern ist ein merklicher Druckabfall nachweisbar. Bei der großen Riepp-Orgel sank er auf $77 \mathrm{~mm}$, bei der Holzhay-Orgel sogar auf $54 \mathrm{~mm}$, an der Lade gemessen. Eine Folge davon ist, daß der Schalldruck beim Zuzug vieler Register weniger ansteigt, als aus der Superposition der Einzelschalldrucke zu erwarten wäre. Derartige Winddruckschwankungen werden vom heutigen Orgelbau als Fehler bewertet und vermieden, obwohl die dadurch hervorgerufenen Frequenzschwankungen sehr wirkungsvoll sind (s. Anm.2).

Die Dispositionen der Orgeln (Registernamen und ihre Fußzahl) sollen hier nicht wiedergegeben werden. Sie sind den genannten Veröffentlichungen $^{5} \mathrm{zu}$ entnehmen.

${ }^{5}$ W. Supper u. H. M e y e r, Barockorgeln in Oberschwaben, Bärenreiterverlag Kassel; J. W ö r s c h i n g, Der Orgelbauer K. Riepp, Rheingoldverlag; J. W ö r s c h i n g, Orgelmonographie 2, Die berühmten Orgelwerke der Abtei Ottobeuren, Rheingoldverlag; H. M e y e r, K. J. Riepp, der Orgelbauer von Ottobeuren, Bärenreiterverlag Kassel.

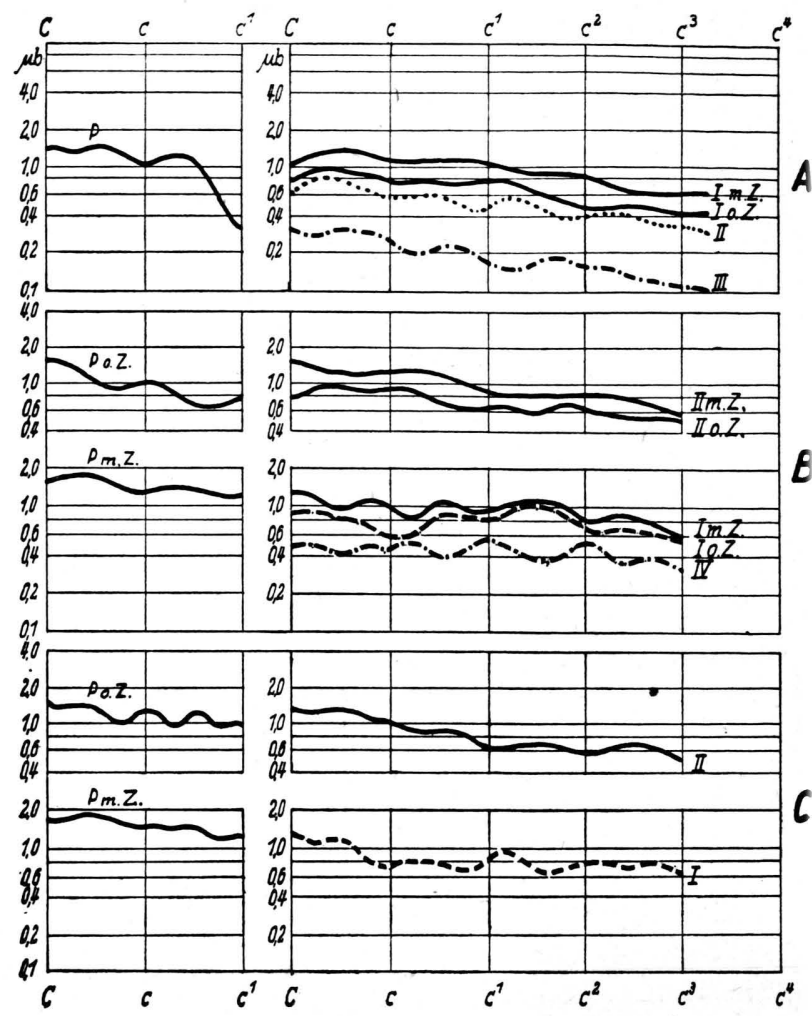

Abb. 1. Schalldrucke der Plenumklänge dreier Barockorgeln.

A: H o l z h a y - Orgel. Pedal (P) : Subbaß 16',

Oktavbaß $8^{\prime}$, Kornettbaß $4^{\prime}+2^{\prime}+1^{3} / 5^{\prime}+1^{1} / 3^{\prime}$.

I. Manual mit Zungen (I m. Z.) : Prinzipal $8^{\prime}+16^{\prime}$, Oktave $4^{\prime}+2^{\prime}$, Mixtur, Trompete $8^{\prime}+4^{\prime}$.

I. Manual ohne Zungen (I o.Z.): dieselben Register wie I m. Z., aber ohne Trompeten.

II. Manual (II): Prinzipal 8', Oktave 4', Sifflöte 2', Quinte 3', Cymbel.

III. Manual (III): Gedackt 8', Spitzflöte 4', Flageolett 2'.

B: Große Ri e p p-Orgel. P o.Z.: Prinzipal 16', Oktave $8^{\prime}$, Copel 16', Quinte 6', Mixtur.

P m. Z.: wie o. Z., aber + Bomba $16^{\prime}$, Trompete $8^{\prime}+4^{\prime}$. Hauptwerk II m. Z.: Copel $8^{\prime}+16^{\prime}$, Prinzipal $8^{\prime}+4^{\prime}$, Quinte $3^{\prime}$, Mixtur, Cymbel, Trompete $8^{\prime}$, Clairon $4^{\prime}$. II o. Z.: dieselben Register wie bei II m. Z., aber ohne Zungenstimmen.

Positiv I m. Z.: Prinzipal 16', Copel 8', Oktave $\mathbf{4}^{\prime}$, Quart 2', Quint $1^{1} / \mathbf{3}^{\prime}$, Fornit, Clairon $4^{\prime}$.

I o. Z.: wie I m. Z., aber ohne Clairon.

Echowerk (IV): Copel 8', Flet 4', Quint 3', Quart 2', Larigo.

C: Kleine Ri ep p-Orgel. P o. Z.: Prinzipal 16', Copel $16^{\prime}$, Flauta $8^{\prime}$, Flet 4', Quint $3^{\prime}$.

P m. Z.: 'wie o. Z., aber + Fagott 8 '.

Hauptwerk II: Copèl $8^{\prime}+16^{\prime}$, Prinzipal $8^{\prime}$, Oktave $4^{\prime}$, Doublet $2^{\prime}$, Mixtur, Cymbel.

Positiv I: Copel 8', Prinzipal 4', Quint 3', Doublet 2', Mixtur. 

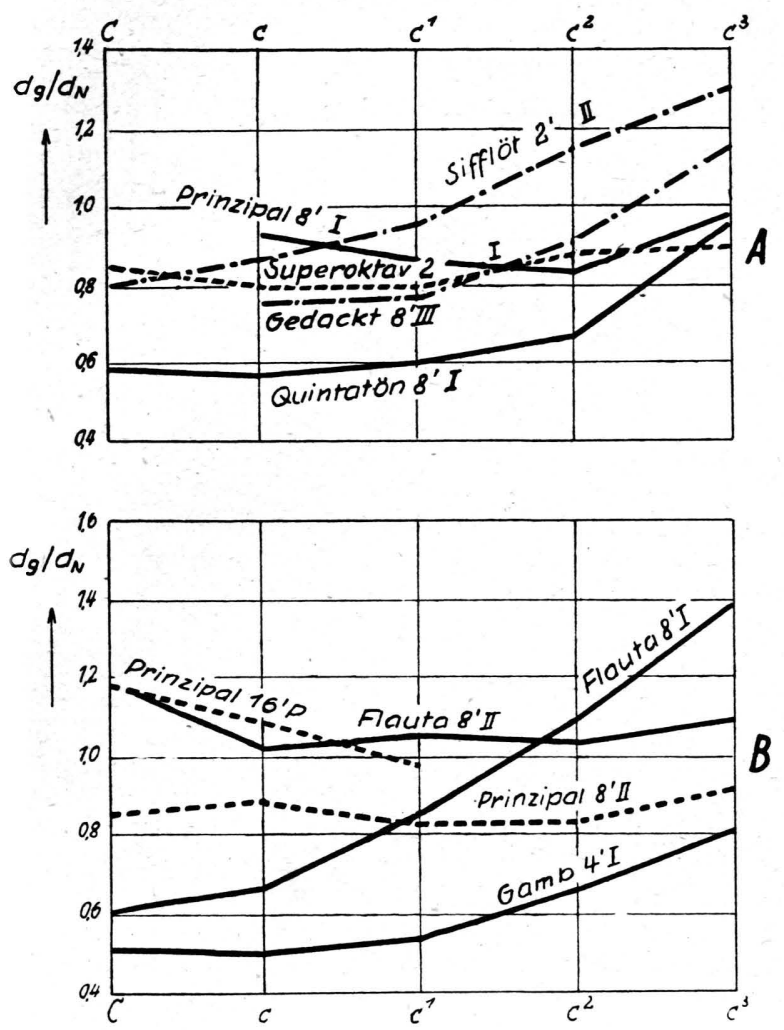

Abb. 2. Mensurkurven als Verhältniswerte der Pfeifendurchmesser $d_{\mathrm{g}}$ (gemessen) $: d_{\mathrm{N}}$ (Töpfersche Normalmensur). A: Holzh a -Orgel, B: Große RieppOrgel.

Auf dieselbe Art wie die Barockorgeln wurden mehrere moderne Orgeln durchgemessen, deren Schalldrucke von Fall zu Fall mitgeteilt werden.

\section{2. $\mathrm{Me} ß \mathrm{~m}$ ethode}

Der Schalldruck wurde mit Kondensatormikrophon, Verstärker und Röhrenvoltmeter, das bei bestimmten Verstärkungsgraden direkt in $\mu \mathrm{b}$ geeicht worden war, gemessen. Das Mikrophon wurde in der Ottobeurener Kirche in $15 \mathrm{~m}$ Entfernung von der untersuchten Orgel an der jeweils gegenüberliegenden Seite des Chorraumes aufgestellt. Dieser Platz war ausgesucht worden, weil die Orgeln symmetrisch in das Chorgestühl am Hochaltar eingebaut sind und ihre Hauptabstrahlung in den Chorraum erfolgt. In Obermarchtal, wo die Orgel in üblicher Weise über dem Hauptportal gegenüber dem Hochaltar steht, befand sich das Mikrophon in $24 \mathrm{~m}$ Entfernung mitten im Schiff. Beim Tönen eines Klanges überlagern sich direkte und von den Wänden reflektierte Wellen derartig, daß sich die bekannten stehenden Wellen ausbilden, bei denen Orte größerer Intensität mit solchen geringerer abwechseln. Diese Erscheinung stört die
Schalldruckmessungen sehr, während das $\mathrm{Ohr}$ bei zweiohrigem Hören darauf weniger empfindlich ist. Auch ist bei der Darbietung von Musik, vor allem solcher polyphonen Charakters, der eingeschwungene Zustand selten. Zur Unterdrückung dieser störenden Einflüsse wurden, wie bei der Lautstärkeuntersuchung von E. Thienhaus und W. Will ms ${ }^{1}$, jeweils drei benachbarte Tasten, also $C$ Cis $D, D i s E F$ usw. gleichzeitig angeschlagen. Dadurch entstehen Klänge mit etwas verschiedenen Wellenlängen, wodurch sich Knoten und Bäuche gleichmäßiger im Raum verteilen. Nach Messung des resultierenden Schalldrucks wurde der abgelesene Mittelwert auf den Schalldruck einer Pfeife bzw. Pfeifengruppe reduziert.

\section{Ergebnisse}

Die gemessenen Schalldrucke sind in den Abb. 1, 3, 4 u. 5 wiedergegeben. Auf der Abszisse ist immer die Tonhöhe in logarithmischem Maß, verdeutlicht durch die Oktaven von $C$, aufgetragen. Innerhalb einer Oktave liegen 4 Meßpunkte. Auf der Ordinate ist, ebenfalls logarithmisch, der Schalldruck aufgezeichnet, wobei meist die Dekade 0,1 bis $1 \mu b$ ausreicht. In manchen Fällen ist der Maßstab etwas verschoben.

Die Ergebnisse allgemeinerer Art seien im folgenden zusammengefaßt:

a) Die Schalldrucke sind auch dann, wenn alle Hauptregister der Orgel gezogen wurden, auffallend gering $(\max .1,8 \mu \mathrm{b})$. Der Unterschied zwischen den leisesten Registern von etwa 0,04 $\mu \mathrm{b}$ und dem genannten Wert beträgt $45: 1$ oder rd. 33 db. Der höchste Schalldruck des Plenums der Hauptwerke beträgt sogar bei Mitwirkung der Zungenpfeifen nur 1,5 $\mu \mathrm{b}$. Ohne diese liegt er bei 1,0 $\mu \mathrm{b}$. Lediglich im Hauptwerk der kleinen Orgel zu Ottobeuren ist er etwas größer $(1,3 \mu b)$. Besonders bemerkenswert ist die Ahnlichkeit der Plenumwerte aller Barockorgeln. Auch der Verlauf längs der Tonskala ist stets schwach abfallend mit wachsender Frequenz auf 0,5 bis $0,6 \mu \mathrm{b}$ bei $c^{\prime \prime \prime}$.

Bei den Pedalwerken erreichen die Maximalwerte 1,8 $\mu \mathrm{b}$. Auch bei diesen ist eine schwach fallende Tendenz zu erkennen. Läßt man die Zungenstimmen fort, dann werden 1,6 bis $0,4 \mu \mathrm{b}$ gemessen. Daß das Pedal der kleinen RieppOrgel, trotzdem es keine gemischten Stimmen besitzt, den geringsten Abfall bei hohen Frequenzen zeigt,wird deutlich, wenn die Schalldrucke von Prinzipal $16^{\prime}$ und Flauta $8^{\prime}$ (Abb. 5 rechts) betrachtet werden. 
Neben den Hauptwerken sind in Abb. 1 die Gesamtwirkungen der anderen Manuale gezeigt. Meist sind diese schwächer und unterscheiden sich außerdem durch ihre Klangfarbe. Besonders abgestuft sind die Kurven der Obermarchtaler Orgel (Abb. 1, A). Dieses Instrument ist, wie erwähnt, ein typischer Vertreter des ausgehenden Barocks und des Übergangs zur Romantik, in welcher Zeit schwache Stimmen beliebt wurden. und ein lautstärkemäßig gestufter Aufbau der Manuale in Aufnahme kam, der sich bis zur Neuzeit erhalten hat.

Bei den Riepp-Orgeln unterscheiden sich Hauptwerk und Positiv viel weniger durch ihre Schalldrucke als durch unterschiedliche Klangfarbe. Nur das 4. Manual der großen Orgel hat schwächere Stimmen. Deutlich ist überall der Zuzug der Zungenstimmen bemerkbar.

Zum Vergleich seien folgende Werte einer modernen Orgel angeführt: Im Hauptwerk: $C=4,0$; $c=2,6 ; c^{\prime}=2,8 ; c^{\prime \prime}=2,3 ; \mathrm{e}^{\prime \prime \prime}=1,4 \mu \mathrm{b}$. Im Pedal: $C=9,0 ; c=7,5 ; c^{\prime}=5,6 \mu b$. Die gezogenen Register waren die gleichen wie bei den Barockorgeln, auch die Zungenstimmen ähnlicher Art waren eingeschaltet. Der subjektive Eindruck solcher Klänge war auffallend laut und wegen des starken Übergewichts tiefer Frequenzgebiete, besonders im Pedal, zu dunkel. Verursacht wurden diese akustischen Eigenschaften vor allem durch zu starken Winddruck (bis $110 \mathrm{~mm}$ ) und zu weite Durchmesser der maßgeblichen Stimmen bei relativ hohen Aufschnitten. Durch diese Maßnahmen wird der Grundton der Pfeifen besonders stark abgestrahlt, was in tiefen Lagen nicht erwünscht ist.

Berücksichtigt man, daß sich beim Zuzug von mehreren Registern die Schalldrucke effektiv addieren, so folgt, daß die angegebenen Werte der Plenumklänge durch die Wirkung der lauten Stimmen zustande kommen. Für die Gesamtwirkung einer Orgel sind deshalb die Prinzipale, Oktaven, Mixturen und starken Zungen: ihr Schalldruckverlauf, ihr Stärkeverhältnis von ausschlaggebender Bedeutung.

Die kleinsten gemessenen Schalldrucke von $0,04 \mu b$ (Abb. 3) sind den örtlichen Störpegelverhältnissen angepaßt. Der Geräuschpegel liegt bei Abwesenheit größerer Störungen, wie Trittschall usw., bei ähnlichen Werten und wird in seiner Höhe meist durch das laufende Gebläse der Orgeln bestimmt. Es hätte darnach nicht viel
Sinn, viel kleinere Schalldrucke als $0,04 \mu \mathrm{b}$ durch eine Stimme der Orgel zu erzeugen, es sei denn, daß der Störpegel durch geeigneten Aufstellungsort des Gebläses und bei besonderen örtlichen Verhältnissen niedriger liegt.

Zusammenfassend ist festzustellen, daß die obere Schalldruckgrenze des Orgelklangs durch eine physiologisch bedingte Empfindungsgrenze, oberhalb derer Klänge und Töne als zu laut beurteilt werden, die untere durch den in der Kirche normalerweise herrschenden Störpegel gegeben ist. Wenn hier gefunden wurde, daß 1,5 bis $1,8 \mu \mathrm{b}$ für die Gesamtwirkung der Orgel günstig ist, so ist daraus $\mathrm{zu}$ schließen, daß auch moderne Orgeln nur mit Winddrucken von 60 bis $80 \mathrm{~mm}$ betrieben zu werden brauchen, sofern nicht ganz außergewöhnliche Verhältnisse vorliegen.

b) Sieht man von der weiter unten besprochenen Welligkeit der Schalldruckdiagramme ab, so kann man Kurven unterscheiden, die mit wachsender Grundtonfrequenz fallen (Mehrzahl), solche, die steigen (Seltenheit) und solche, die ungefähr gleiches Niveau behalten. Werden die Mensurkurven der Abb. 2 mit den Schalldruckkurven der Abb. 3, 4 u. 5 verglichen, so ergibt sich, daß der Schalldruck und sein Verlauf von der Pfeifenmensur abhängt. Damit diese Beziehung deutlich wird, sind, zum Unterschied gegen die sonst übliche Darstellung, die Mensurkurven als Verhältniswerte von gemessenem Durchmesser $d_{\mathrm{g}} \mathrm{zu}$ dem der sogen. Normalmensur $d_{N}$ von Töp for ${ }^{6}$ angegeben. Daraus ist zu ersehen: 1. Je größer das angeführte Verhältnis der Durchmesser ist, um so größer ist der abgegebene Schalldruck. 2. Steigt das Verhältnis stark an, dann bleibt der Schalldruck im ganzen Bereich nahezu konstant

${ }^{6}$ Die Töpfersche Mensur wurde dem umfassenden Werk von Ch. M a h r e n o lz, Die Orgelregister, Bärenreiterverlag Kassel, entnommen (S. 14).

$$
\text { Erläuterung zu Abb. } 3 \text {. }
$$

Zusammensetzung der Mixtur:

$$
\begin{aligned}
& C=2^{\prime}+1^{1} / 3^{\prime}+1^{\prime}+2 / 3^{\prime}+1 / 2^{\prime} \\
& c^{\prime}=4^{\prime}+2^{2} / 3^{\prime}+2^{\prime}+1^{1} / 3^{\prime}+1^{\prime} \\
& c^{\prime \prime}=4^{\prime}+4^{\prime}+2^{2} / 3^{\prime}+2^{\prime}+1^{1} / 3^{\prime},
\end{aligned}
$$

der Sesquialter: $2^{2} / 3^{\prime}+2^{\prime}$, des Kornett: $2^{2} / 3^{\prime}+2^{\prime}+1^{3} / 5^{\prime}$, der Cymbel:

$$
\begin{aligned}
& C=2^{\prime}+1^{1} / 3^{\prime}+1^{\prime}+2 / 3^{\prime}+1 / 2^{\prime}, \\
& c^{\prime}=4^{\prime}+2^{2} / 3^{\prime}+2^{\prime}+1^{1} / 3^{\prime}+1^{\prime}, \\
& c^{\prime \prime}=4^{\prime}+4^{\prime}+2^{2} / 3^{\prime}+2^{2} / 3^{\prime}+2^{\prime},
\end{aligned}
$$

des Kornett: $2^{2 / 3^{\prime}}+2^{\prime}+1^{3} / \mathbf{s}^{\prime}$,

des Hörnle: $2^{\prime}+13 / 5^{\prime}$,

des Kornettbaß: $4^{\prime}+2^{2} / 3^{\prime}+2^{\prime}+1^{3} / 5^{\prime}$.

(Alle Angaben nach W. Supper und H. Meyer ${ }^{5}$.) 

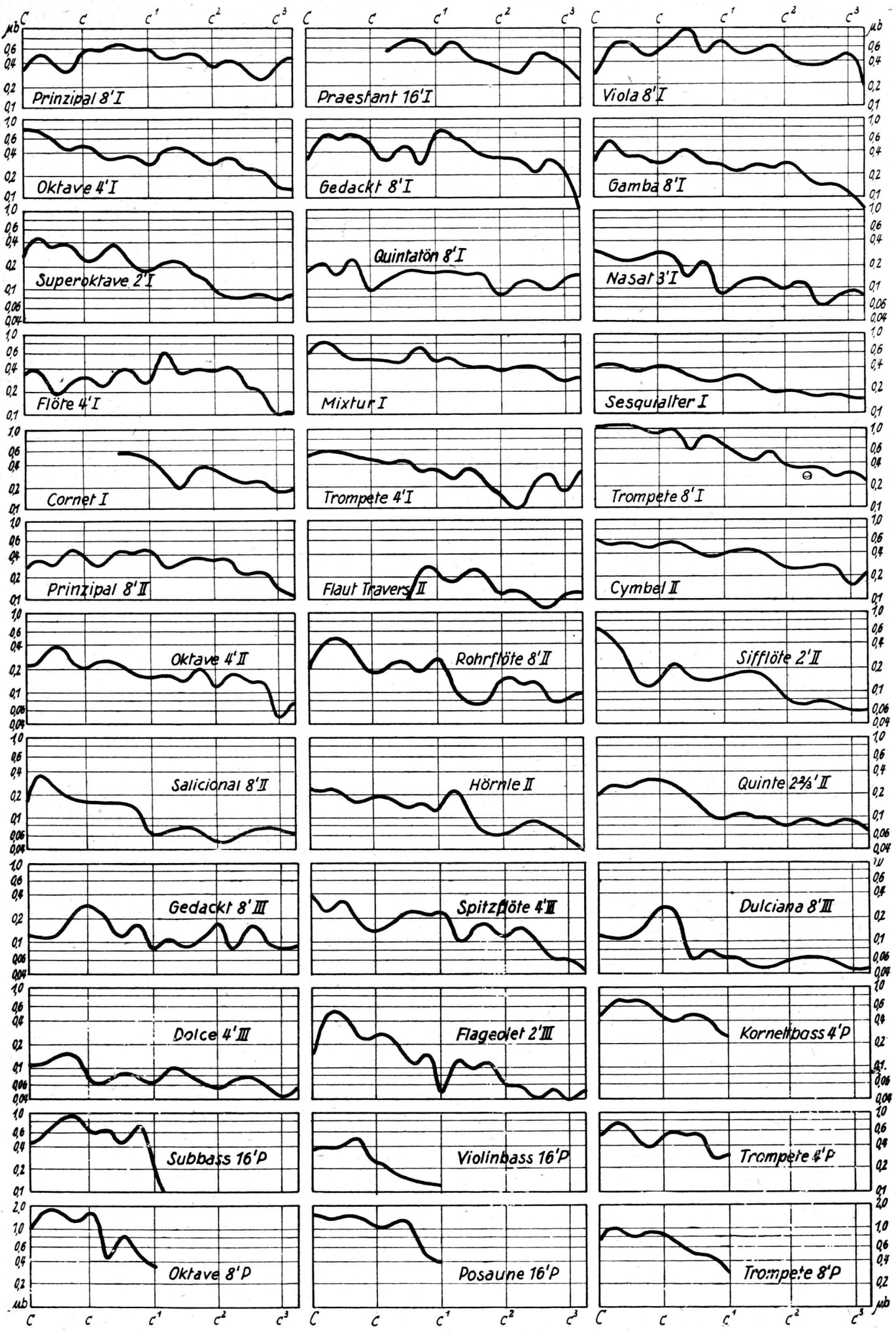

Abb. 3. Schalldruckkurve der Hol zh a y-Orgel. Erläuterung s. S. 302 . 
(Abb. 3 Gedackt 8' III ${ }^{7}$, Quintatön 8' I, Abb. 4 Flauta $8^{\prime}$ I). Bei sehr hohen Frequenzen macht sich eine Erhöhung der Weite nicht im gleichen Maß geltend (Abb. 3 Sifflöte 2'). Ist das Verhältnis $d_{\mathrm{g}}: d_{\mathrm{N}}$ im Tonbereich konstant, dann fällt der Schalldruck (Abb. 3 Superoktav 2', Abb. 4 Flauta $8^{\prime}$ II, Prinzipal 8' II). Nimmt $d_{g}: d_{N}$ ab, so fällt der Schalldruck natürlich ebenfalls (Abb. 4 Prinzipalbaß $16^{\prime}$ ).

Engmensurierte Stimmen, wie Gamben, Quintatön, Salicional (Salicet), liefern schwache, weite Stimmen starke Klänge, falls nicht irgendwelche Maßnahmen am Pfeifenfuß usw. getroffen wurden, um den Klang abzuschwächen. In den Hauptwerken sind die Prinzipale und Oktaven mit weiteren Mensuren versehen als in den Nebenwerken; deshalb sind die Schalldrucke bei ersteren größer (Abb. 3 Prinzipale 8' I u. II sowie die Oktaven I u. II).

c) Zungenpfeifen haben ihr Schalldruckmaximum meist in den tiefen Lagen. Sie sind besonders laut, wenn sie weite Becher, wie z. B. Trompete, Bombarde und Clairon, haben; sie übertreffen in den tiefen Lagen alle anderen Register der Orgel (Abb. 3 u. 4). Dadurch sind sie besonders geeignet zur letzten Steigerung des Gesamtklanges. Ihre charakteristische Wirkung verdanken sie außer ihrer Lautstärke besonders ihrem großen Reichtum an Obertönen.

d) Da die Meister des Barocks in den tiefen Lagen relativ engere Mensuren als in den anderen anwandten, wird die Obertönigkeit der Baßpfeifen erhöht, die der Diskantpfeifen verkleinert. Durch dieses Vorgehen, das besonders bei der HolzhayOrgel zu beobachten ist, wird eine wesentliche Voraussetzung zur Erzeugung hervorragender Klänge, nämlich die Betonung der Mittellage, erfüllt ${ }^{2}$. Die Riepp-Orgeln besitzen etwas größere Schalldrucke in den tiefen Lagen. Dieser Unterschied ist auch subjektiv festzustellen; allerdings ist es möglich, daß spätere Erhöhung der Aufschnitte diese Wirkung verstärkt hat. Jedenfalls wirken die Riepp-Orgeln in den tiefen Lagen voller und dunkler.

e) Obertonarme Register, wie Copeln und Flöten, zeigen in ihrem Schalldruckverlauf auffallende Intensitätsschwankungen. Diese Erscheinung ist besonders erkennbar bei den RieppOrgeln (Abb.4). Die Welligkeiten, die trotz eines kontinuierlichen Mensurverlaufs vorhanden sind,

7 Die römischen Ziffern bezeichnen das Manual. haben verschiedene Ursachen. Einmal tritt eine Rückwirkung des Raumes auf die schwingenden Pfeifen auf. Die Pfeifen tönen dann am besten, wenn sie in einem Schwingungsbauch der stehenden Welle stehen. Andere Pfeifen, die sich an Knotenstellen befinden, tönen leiser. Diese Erscheinungen treten natürlich um so deutlicher auf, je obertonärmer eine Pfeife ist. Strahlt sie mehrere Teiltöne $a b$, so kann wohl für einen der ungünstige Fall der Knotenstelle eintreten, für die anderen trifft dies mit großer Wahrscheinlichkeit nicht zu. Die obertonreichen Register, wie die Zungenpfeifen und gemischten Stimmen der Mixturen und Cornette, zeigen auch aus diesem Grunde die geringste Welligkeit in ihrem Schalldruckverlauf. Zu dieser ,,äußeren“ Beeinflussung des Schwingungszustandes kommt eine „,innere“. Bei der Schleiflade treten nämlich Rückwirkungen der Kanzellenräume auf die Pfeifenschwingungen auf, so daß eine Pfeife dann am lautesten tönt, wenn beide Räume, die der Kanzelle und der Pfeife, in Resonanz sind ${ }^{8}$.

Es kommt noch hinzu, daß sich bei den Messungen in der Ottobeurener Kirche einige Meter hinter dem Mikrophon Wand und Sitze des Chorgestühls befanden. Dadurch war die Ausbildung stehender Wellen sehr begünstigt, die auch durch die 3 benachbarten Frequenzen nicht völlig unterdrückt wurden.

Besonders eigenartig ist, daß das Ohr die gezeigten Schalldruckschwankungen nicht als fehlerhaft empfindet. Abwechslungen des Schall-

$8 \mathrm{~K}$. J un g, Die abgestimmte Tonkanzelle, Instrumentenbau-Z. 1931 u. 1932.

Erläuterung.zu A bb. 4.

Zusammensetzung der Fornit (Fourniture):

$$
\begin{aligned}
& C=1^{\prime}+2 / 3^{\prime}+2 / 3^{\prime}+1 / 2^{\prime}+1 / 3^{\prime} \\
& c=2^{\prime}+1^{1} / 3^{\prime}+1^{\prime}+2 / 3^{\prime}+1 / 2^{\prime} \\
& c^{\prime}=2^{2} / 3^{\prime}+2^{\prime}+1^{1} / 3^{\prime}+1^{1} / 3^{\prime}+1^{\prime} \\
& c^{\prime \prime}=4^{\prime}+2^{2} / 3^{\prime}+2^{\prime}+1^{1} / 3^{\prime}+2^{\prime}+1^{1} / 3^{\prime} \\
& c^{\prime \prime \prime}=4^{\prime}+2^{2} / 3^{\prime}+2^{\prime}+4^{\prime}+2^{2} / 3^{\prime}+2^{\prime},
\end{aligned}
$$

der Mixtur II:

$$
\begin{aligned}
& C=2^{1} / 3^{\prime}+2^{\prime}+1^{1} / 3^{\prime}+1^{\prime} \\
& c^{\prime}=4^{\prime}+2^{2} / 3^{\prime}+2^{\prime}+1^{1} / 3^{\prime} \\
& c^{\prime \prime}=5^{1} / 3^{\prime}+4^{\prime}+2^{2} / 3^{\prime}+2^{\prime} \\
& c^{\prime \prime \prime}=8^{\prime}+5^{1} / 3^{\prime}+4^{\prime}+2^{2} / 3^{\prime},
\end{aligned}
$$

des Kornett II: $8^{\prime}+4^{\prime}+2^{2} / 3^{\prime}+2^{\prime}+1^{3} / 5^{\prime}$, der Cymbel:

$$
\begin{aligned}
& C=1^{\prime}+2 / 3^{\prime}+2 / 3^{\prime}+1 / 2 \\
& c=1^{1} / 3^{\prime}+1^{\prime}+2 / 3^{\prime}+2 / 3^{\prime}+1 / 2^{\prime} \\
& c^{\prime}=2^{2} / 3^{\prime}+2^{\prime}+1^{1} / 3^{\prime}+1^{\prime}+1^{\prime} \\
& c^{\prime \prime}=5^{1} / 3^{\prime}+4^{\prime}+2^{2} / 3^{\prime}+2^{\prime}+1^{1} / 3^{\prime}+1^{1} / 3^{\prime} \\
& c^{\prime \prime \prime}=5^{1} / 3^{\prime}+4^{\prime}+2^{2} / 3^{\prime}+2^{2} / 3^{\prime}+2^{\prime}+2^{\prime},
\end{aligned}
$$

des Kornett (Resi=Recit) III: $8^{\prime}+4^{\prime}+2^{2} / 3^{\prime}+2^{\prime}+1^{3} / 5^{\prime}$, der Pedalmixtur: $2^{2} / 3^{\prime}+2^{\prime}+11^{1} / 3^{\prime}$. 

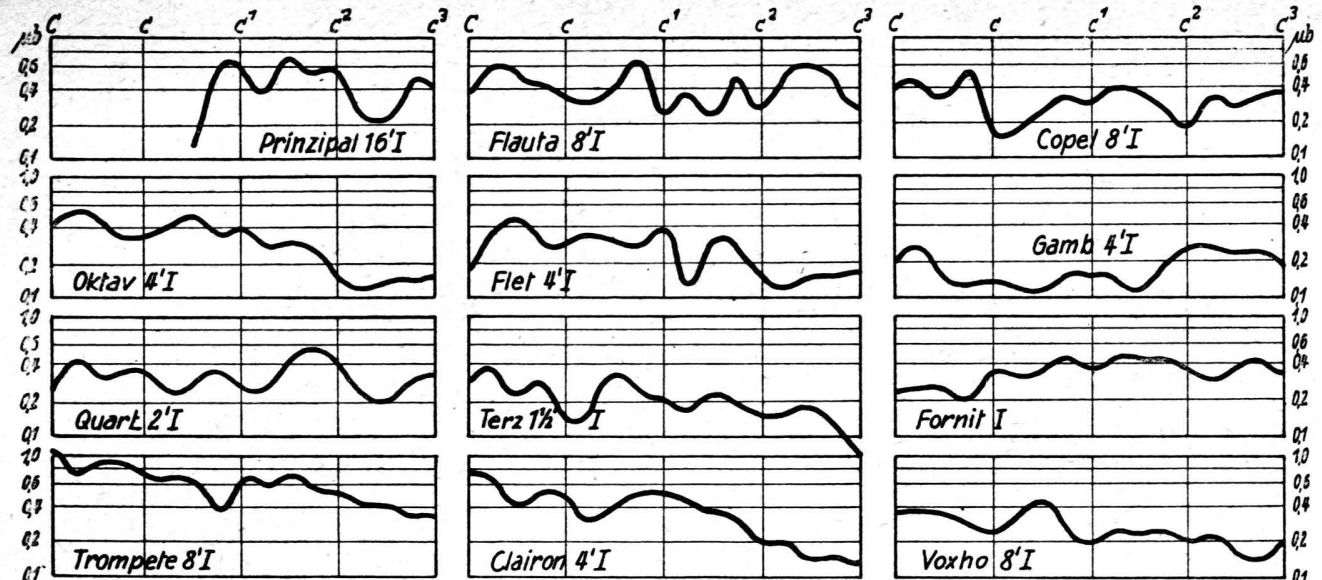

Trompere 8'I
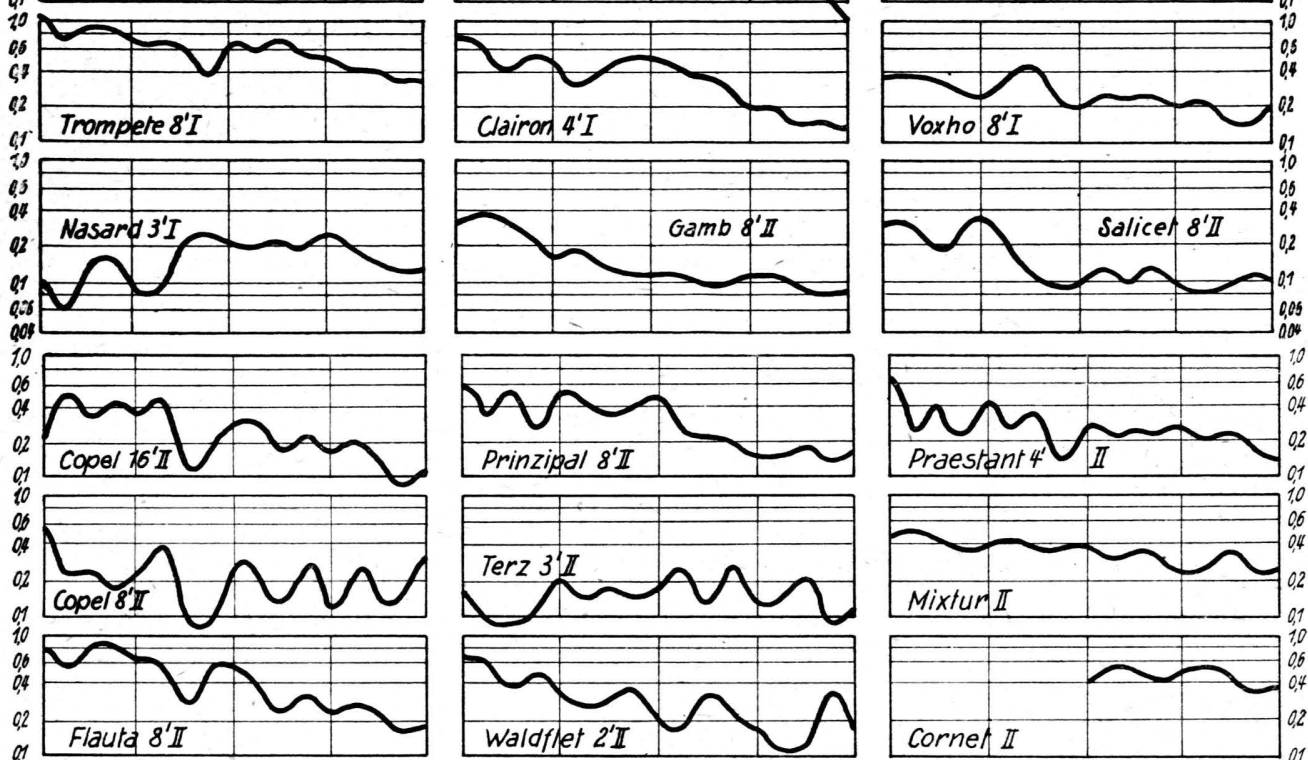

Q.2 Flauta 8 'II
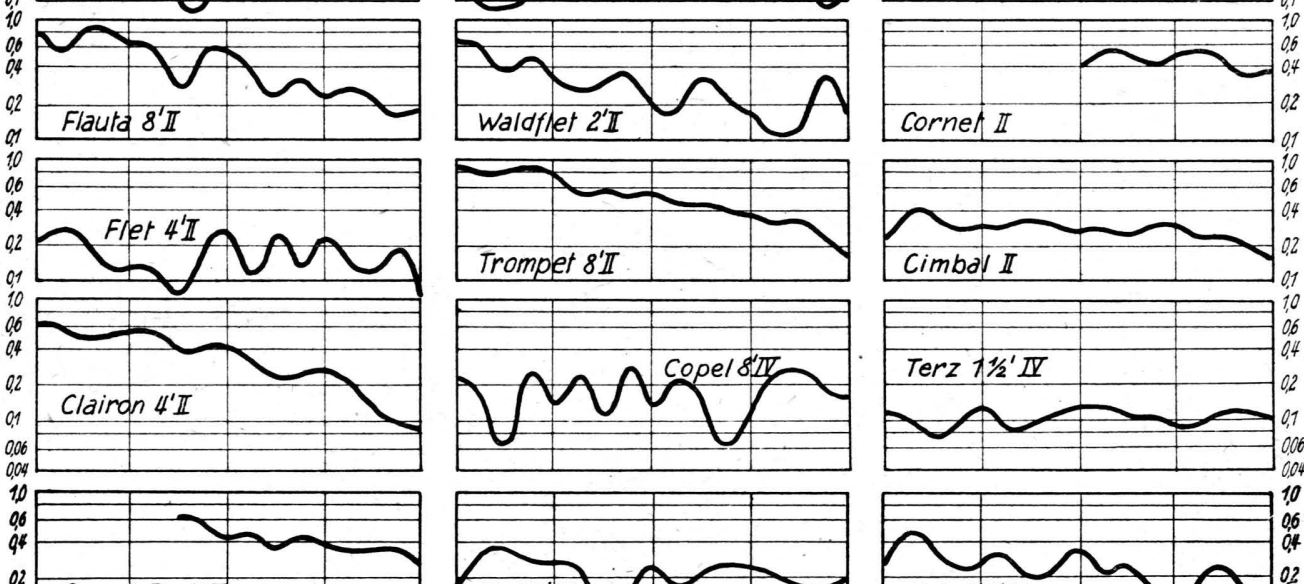

Cornet Recir III
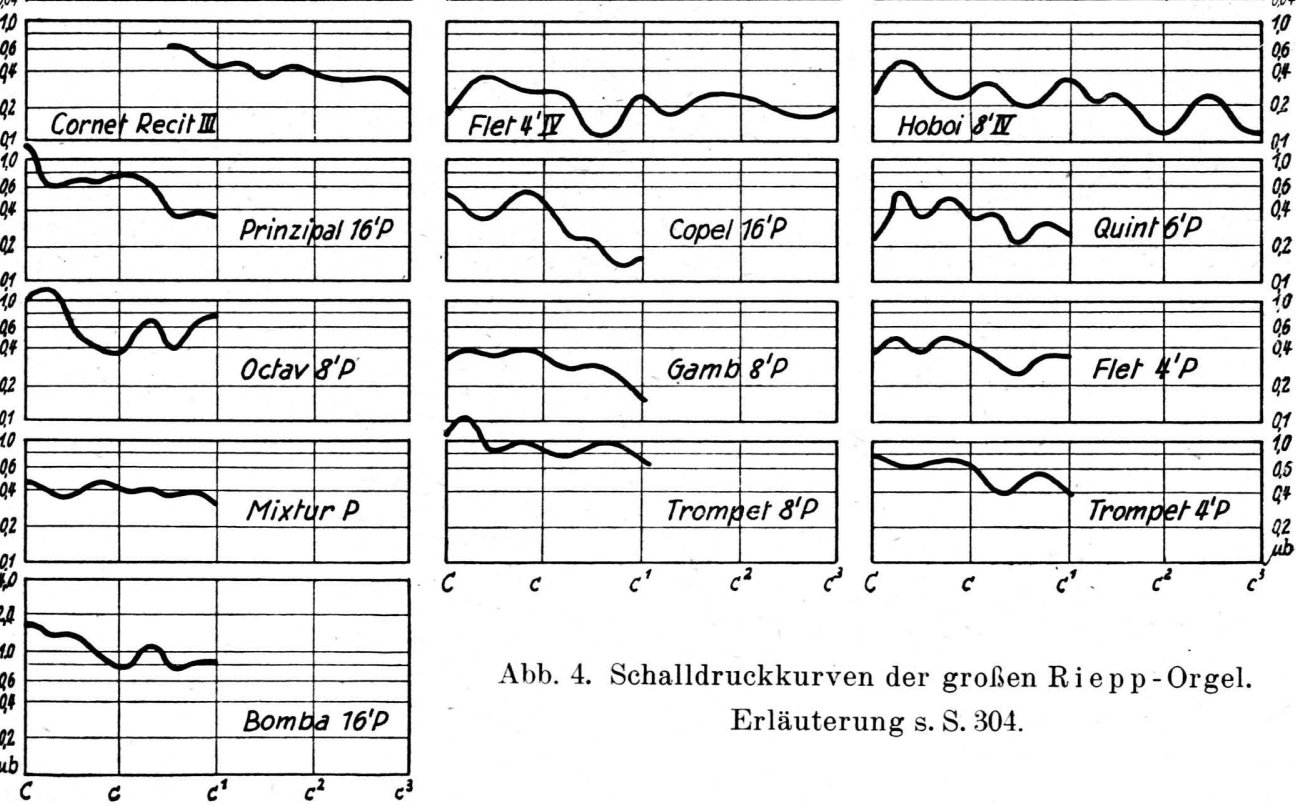

Abb. 4. Schalldruckkurven der großen Ri e p p-Orgel. Erläuterung s. S. 304. 

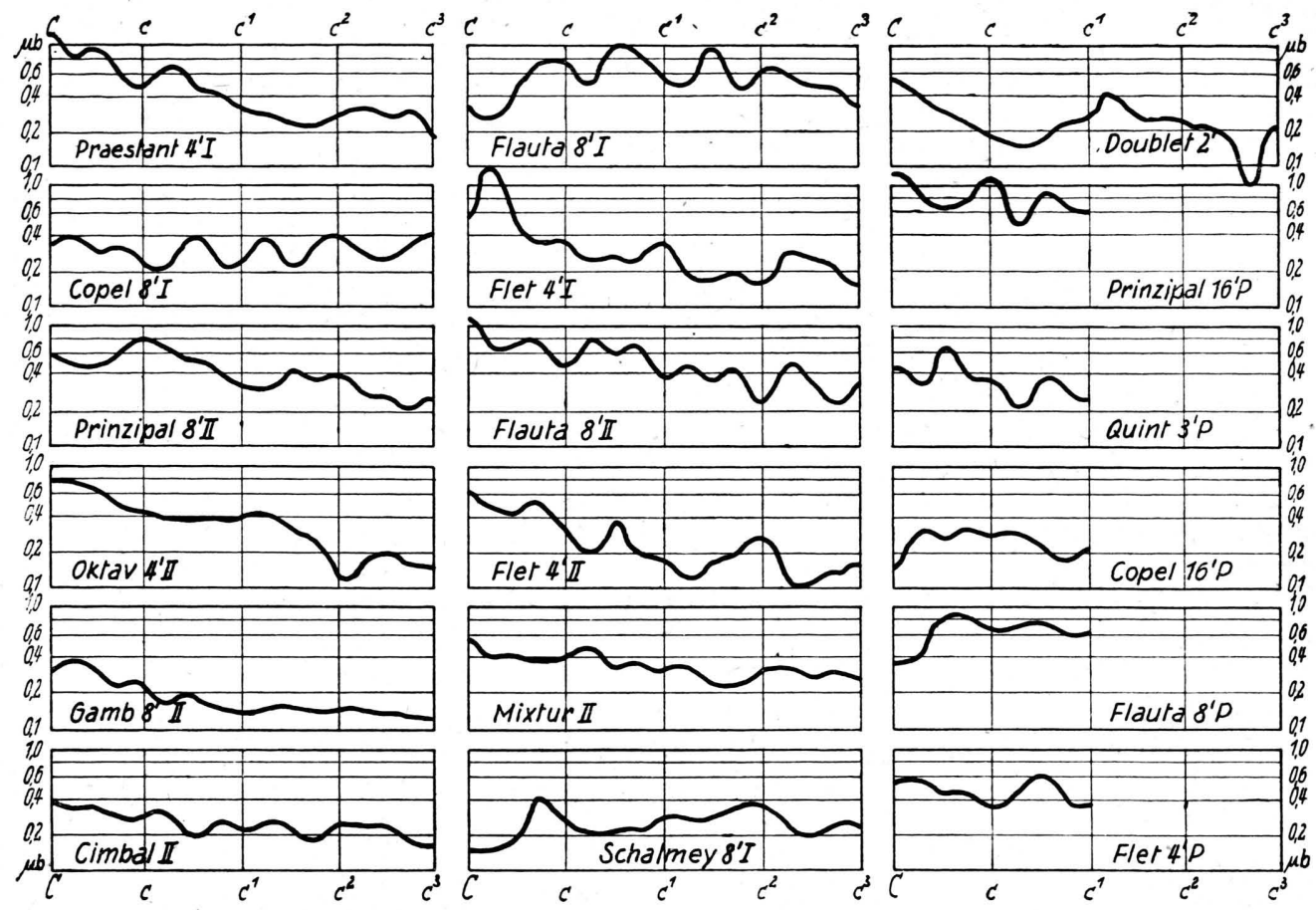

Abb. 5. Schalldruckkurven der kleinen Riepp-Orgel.

Zusammensetzung der Mixtur II:

$$
\begin{aligned}
& C=1^{1} / 3^{\prime}+1^{\prime}+2 / 3^{\prime}+1 / 2^{\prime} \\
& c=2^{2} / 3^{\prime}+2^{\prime}+1^{1} / 3^{\prime}+1^{\prime} \\
& c^{\prime}=4^{\prime}+2^{2} / 3^{\prime}+2^{\prime}+1^{1} / 3^{\prime} \\
& c^{\prime \prime}=5^{1} / 3^{\prime}+4^{\prime}+2^{2} / 3^{\prime}+2^{\prime} \\
& c^{\prime \prime \prime}=5^{1 /} / 3^{\prime}+4^{\prime}+2^{2} / 3^{\prime}+2^{\prime},
\end{aligned}
$$

der Cymbel II:

$$
\begin{aligned}
& C=2 / 3^{\prime}+1 / 2^{\prime}+1 / 3^{\prime} \\
& c=1^{\prime}+2 / 3^{\prime}+1 / 2^{\prime} \\
& c^{\prime}=11^{1} / 3^{\prime}+1^{\prime}+2 / 3^{\prime} \\
& c^{\prime \prime}=2^{2} / 3^{\prime}+2^{\prime}+1^{1} / 3^{\prime} \\
& c^{\prime \prime \prime}=4^{\prime}+2^{2} / 3^{\prime}+2^{\prime} \text {. }
\end{aligned}
$$

(Alle Angaben nach J. Wö r s ch i n g ${ }^{5}$.)

drucks längs der Tonskala wirken sich, sofern sie in den gezeigten Grenzen bleiben, nicht ungünstig aus. Außerdem ist dabei noch zu berücksichtigen, daß bei Kombination solcher Register mit anderen ein Ausgleich des Gesamtschalldrucks eintritt. Die Obertonzusammensetzung wechselt trotzdem längs der Tonskala. Denkt man sich z. B. eine Tonfolge von $C$ ab aufwärts mit den Registern Gedackt 8', Spitzflöte 4' und Flageolett 2' im III. Manual der 'Holzhay-Orgel gespielt, so folgt aus den Schalldruckaufnahmen, daß zunächst das Gedackt und Flageolett hinter der Wirkung der Spitzflöte zurückstehen. Von Dis ab wird aber das Flageolett stark. Bei $c$ überwiegt Gedackt, während die Spitzflöte schwächer ist als Flageolett. Bei $c^{\prime \prime}$ liefert die Spitzflöte mehr Schalldruck als die anderen, und bei $c^{\prime \prime \prime}$ überwiegt Gedackt. So wechselt der Klang der Kombination von Lage zu Lage. Daß er dabei immer von schönster Wirkung ist, zeugt von dem hervorragenden Geschick des Orgelbauers.
Ohne auf jede Einzelheit der Register (Abb. 3, 4 u. 5) einzugehen, sei im folgenden auf einige Eigentümlichkeiten hingewiesen. Bei der HolzhayOrgel fällt vor allem die günstige Schalldruckverteilung der Prinzipale und Oktaven auf. Während Prinzipal $8^{\prime}$ in der großen Oktave verhältnismäßig schwach ist, überwiegt in diesem Frequenzbereich die Oktave $4^{\prime}$, so daß bei der Kombination beider der Grundton weniger stark ist. Die Gefahr, daß die Klänge dumpf wirken, ist also vermieden. In den höheren Lagen wächst die Intensität des Prinzipals, deshalb wirken diese Klänge voll. Der Abfall der Superoktave 2' wirkt sich ebenfalls günstig aus, weil dadurch eine zu helle Klangfarbe nicht auftreten kann. Etwas anders ist das Verhalten der entsprechenden Register bei Riepp. Die tiefen Lagen sind stärker, und Prinzipal $8^{\prime}$ überwiegt in der großen und kleinen Oktave. Allerdings kehrt sich das Verhältnis in der eingestrichenen Oktave um. Diese Klänge sind deshalb heller als bei Holzhay. Be- 
sonders ist auf das Verhalten dieser Stimmen im Positiv der großen Riepp-Orgel zu achten. Von der eingestrichenen Oktave nach oben ist Quart $2^{\prime}$ (entspricht der Superoktave 2') sehr stark. Die Klänge des Positivs sind also besonders hell. Riepp hat es verstanden, trotz ähnlicher Intensität (Abb.1) die Klangfarbe von Hauptwerk und Positiv deutlich voneinander abzusetzen. Ein ganz ähnliches Verhalten zeigt auch die kleinere Orgel, bei der in den höheren Lagen Doublet $2^{\prime}$ relativ stärker ist als $8^{\prime}$ und 4'. Im Hauptwerk dieser Orgel finden ähnliche Überschneidungen statt wie bei Holzhay. Insgesamt ist aus den Kurven dieser Register zu entnehmen, daß das Plenum Holzhays ausgeglichener klingen wird als das von Riepp. Während ersterer eine optimale Klangwirkung in allen Lagen anstrebt, weisen die Werke Riepps eine größere Mannigfaltigkeit auf, indem die tiefen Lagen dunkel, die hohen Lagen hell klingen. Dieses Verhalten ist auch subjektiv wahrzunehmen. Man erkennt, daß innerhalb der Grenzen sehr gut wirkender Schalldruckverteilungen viele mögliche Variationen liegen.

Beim Vergleich der Prinzipale beider Meister fällt noch auf, daß diese bei Riepp verhältnismäßig leise sind. Sie werden sogar meist von den Flöten übertroffen, was daher rührt, daß diese Register teilweise auch im Prospekt stehen und die Prinzipale oft erheblich an Weite übertreffen. Beispiele dafür sind Prinzipal $8^{\prime}$ und Flauta $8^{\prime}$ im Hauptwerk der großen Orgel. Ersterer liefert maximal 0,6 $\mu \mathrm{b}$ bei einem Durchmesserverhältnis von 0,85 , Flauta $8^{\prime}$ liefert maximal $0,87 \mu b$ bei $d_{\mathrm{g}}: d_{\mathrm{N}}=1,2$. In der Tab. 1 sind einige Schalldruckwerte moderner Orgeln mitgeteilt.

Man ersieht daraus, daß zwar die notwendige Schalldruckstufung der Stimmen untereinander

\begin{tabular}{|c|c|c|c|c|c|}
\hline Register & $C$ & $c$ & $c^{\prime}$ & $c^{\prime \prime}$ & $c^{\prime \prime \prime}$ \\
\hline $\begin{array}{l}\text { Prinzipal } 16^{\prime} \\
\text { Prinzipal } \delta^{\prime} \\
\text { Oktave } 4^{\prime} \\
\text { Oktave } 2^{\prime}\end{array}$ & $\begin{array}{l}1,0 \\
1,2 \\
0,6 \\
0,36\end{array}$ & $\begin{array}{l}1,5 \\
1,3 \\
0,7 \\
0,28\end{array}$ & $\begin{array}{l}0,7 \\
0,9 \\
0,4 \\
0,21\end{array}$ & $\begin{array}{l}0,7 \\
0,8 \\
0,6 \\
0,26\end{array}$ & $\begin{array}{l}0,5 \\
0,6 \\
0,35 \\
0,18\end{array}$ \\
\hline $\begin{array}{l}\text { Prinzipal } 8^{\prime} \\
\text { Oktave } 4^{\prime} \\
\text { Oktave } 2^{\prime}\end{array}$ & $\begin{array}{l}1,2 \\
1,8 \\
1,4\end{array}$ & $\begin{array}{l}1,2 \\
1,0 \\
0,9\end{array}$ & $\begin{array}{l}1,5 \\
0,9 \\
0,8\end{array}$ & $\begin{array}{l}0,6 \\
0,8 \\
0,5\end{array}$ & $\begin{array}{l}0,8 \\
0,9 \\
0,45\end{array}$ \\
\hline $\begin{array}{l}\text { Prinzipal } 8^{\prime} \\
\text { Oktave } 4^{\prime} \\
\text { Oktave 2 } 2^{\prime} \\
\text { Prinzipal } 16^{\prime}\end{array}$ & $\begin{array}{l}1,4 \\
2,0 \\
2,0 \\
3,0\end{array}$ & $\begin{array}{l}1,5 \\
1,2 \\
0,9 \\
1,3\end{array}$ & $\begin{array}{l}1,9 \\
0,95 \\
0,8 \\
1,6\end{array}$ & $\begin{array}{l}0,65 \\
0,7 \\
0,5 \\
1,9\end{array}$ & $\begin{array}{r}0,6 \\
0,4 \\
0,5 \\
0,7\end{array}$ \\
\hline
\end{tabular}

Tab. 1. Schalldruckwerte moderner Orgeln in $\mu b$. vorhanden ist, daß aber alle Schalldrucke, insbesondere die der tiefen Lagen, zu hoch sind, so daß die Orgeln zu laut und dunkel klingen. Allerdings wurden bei einem Instrument Werte gemessen, die denen der Barockorgeln nahezu gleichkamen, und es ist eine gute Bestätigung der Allgemeingültigkeit der mitgeteilten Werte, daß in diesem Fall eine besonders günstige Wirkung der Orgel erzielt wurde.

Im Plenum der Orgel spielen die gemischten Stimmen, wie Mixturen, Fornit (Fourniture) und Cimbeln, eine große Rolle. Wenn auch ihre Bedeutung in der Erzeugung künstlicher Obertöne in ganz bestimmten Frequenzbereichen liegt, wodurch das Klangspektrum des Plenums seine wirksame Form erhält, so ist ihre Gesamtintensität vor allem im Verhältnis zu den anderen Stimmen wichtig. Am stärksten unter diesen Registern ist die Mixtur im Hauptwerk der HolzhayOrgel, die maximal 0,8 $\mu \mathrm{b}$ abgibt. Die Cymbel ist dagegen viel schwächer. Besonders schwach sind die gemischten Stimmen von Riepp, die maximal nur $0,5 \mu b$ erzeugen. Der Intensitätsabfall mit wachsender Frequenz ist bei diesen Stimmen gering, weil sich die Durchmessermensuren in den oberen Lagen stark erweitern.

Stimmen besonders eigenartiger Wirkung sind die Cornette, die sich ebenfalls aus mehreren Pfeifen zusammensetzen, unter denen die Terz als künstlicher Oberton eine besondere Rolle spielt. Da die Mensur meist recht weit ist, sind die Schalldrucke entsprechend groß. Diese Stimmen sind als Soloregister gedacht und werden, falls sie nicht selbst die Grundtonlage enthalten, zusammen mit einem 8'-Register gebraucht. Auch das Hörnle von Holzhay gehört zu den Cornetten. Es ist aus zwei Pfeifenreihen $2^{\prime}$ und $1^{3 / 5}$ zusammengesetzt. Da sein Schalldruck in der eingestrichenen Oktave stark abfällt, ist seine Wirkung sehr dezent. Zu den solistisch gebrauchten Registern sind auch Quintatön, Salicional, Gamben u. ä. zu zählen, die wegen ihrer engen Mensur schwache Klänge liefern. Eine Ausnahme davon macht die Viola von Holzhay, die in den meisten Lagen runde, flötenartige Klangwirkungen hervorbringt.

Besonders verschönt werden die Barockorgeln durch ihren reichen Gehalt an Aliquotstimmen, wie z. B. Quinten und Terzen, welche eigentümliche Klangmischungen herzustellen gestatten. Diese Register sind meist viel schwächer als die Prinzipale und Oktaven (maximal $0,4 \mu \mathrm{b}$ ). Bei 
Holzhay haben sie in den höheren Oktaven nur geringe Intensität, bei Riepp liegen sie in den gleichen Frequenzgebieten über $0,1 \mu \mathrm{b}$ und färben deshalb stärker.

Da beide Orgelbauer ihre Copeln bzw. Gedackten mit stark veränderlicher Weite bauten, nämlich in der Tiefe eng, in der Mitte als Rohrflöte und in der Höhe offen, so erhält man bei großer Variation der Klangfarbe einen fast gleichen Druckverlauf längs der Tonskala. Die Flöten der Orgeln sind von ganz besonderem Wohllaut. Sie werden meist als Rohrflöten, aber auch gedeckt oder konisch gebaut. Ihre Mensur ist in den Hauptwerken weit, so daß eine ziemlich beträchtliche Schalldruckabgabe zustande kommt. Meist erweitern sie sich in der Höhe stark und klingen deshalb auch dort laut. Durch ihre große Weite bei relativ hohem Aufschnitt wird besonders der Grundton abgestrahlt, wodurch der Klang Rundung und Fülle bekommt.

Die Register der Pedale sind gegenüber denen der modernen Orgel besonders schwach. Bei der großen Riepp-Orgel sind die Werte der tiefsten Lagen von Prinzipal $16^{\prime}$ bemerkenswert, weil die zugehörigen Pfeifen in einem eigenen Prospekt stehen und besonders günstige Abstrahlungsbedingungen haben. Außerdem sind die Pfeifen mit verstärkten Windzuführungen versehen. Man erkennt deutlich den Abfall nach hohen Frequenzen von 1,3 $\mu \mathrm{b}$ ab. Die Prinzipalbässe der modernen Orgeln zeigen dagegen z. B. folgende Schalldrucke: $C=3,2 ; \quad c=1,8 ; \quad c^{\prime}=1,2 ; \quad$ oder $\quad C=5 ; \quad c=3,2$; $c^{\prime}=1,7 \mu b$. Derartige Bässe wirken zu stark und vor allem dann sehr unschön, wenn die Grundtöne im Klang überwiegen. Die andern Register der Pedale sind meistens leiser. Die Pedalmixtur der großen Riepp-Orgel ist verhältnismäßig schwach. Auch bei fehlender Grundtonstärke werden die Baßtöne hervorgehoben durch die Zungenstimmen: Bombarde, Posaune und Trompete, welche durch ihren starken Obertongehalt wegen des nichtlinearen Arbeitens des Ohres eine starke Grundtonempfindung erzeugen. Dem gleichen Zweck dient auch die Quint $6^{\prime}$, die mit der Oktave $8^{\prime}$ den Differenzton in der $16^{\prime}$-Lage erzeugt. Die Zungen gleichen Namens geben bei der modernen Orgel wesentlich mehr Schalldruck. Dort wurde gemessen: bei Posaune $16^{\prime}: C=3,6$; $c=2,2 ; c^{\prime}=0,7$, oder sogar $C=4,5 ; c=2,6$ und $c^{\prime}=1,0 \mu \mathrm{b}$. Bei den Trompeten ist der Unterschied ähnlich. Die Wirkung solch starker Register ist auf den Zuhörer zwar erheblich, aber weniger schön.

Die schwächeren Zungenstimmen von Riepp sind einzigartig und ganz hervorragend. Die Vox humana seiner großen Orgel ist bekannt und berühmt. Alle diese Stimmen, wie Cromor (Krummhorn), Schalmey usw., liefern geringe Schalldrucke, naturgemäß aber sehr obertonreiche Klänge. Die Zungenstimmen der Holzhay-Orgel sind alle modern bzw. in neuerer Zeit ergänzt worden. Ihr Druckverlauf ist durchaus denen originaler Zungenpfeifen ähnlich, so daß sie sich den alten Stimmen gut anpassen.

Die Untersuchungen wurden ermöglicht durch die freundliche Unterstützung des Bischöfl. Ordinariats in Rottenburg, des Caecilienvereins und der Fa. E. F. W a l c k e r \& Cie. in Ludwigsburg. Hr. Prof. $\mathrm{K}$ o s s e l stellte mir bereitwilligst die Hilfsmittel seines Instituts zur Verfügung. Allen Genannten und Ungenannten, die aus Liebe zur Sache zum Gelingen beitrugen, danke ich hiermit herzlich.

\title{
Zur Theorie der Streuung langsamer Neutronen an freien Protonen (Berichtigung)
}

\author{
Von ERICH HÜCKeL ${ }^{1}$ \\ Aus der theoretischen Abteilung des Physikalischen Instituts der Universität Marburg \\ (Z. Naturforschg. 3 a, 308-309 [1948]; eingegangen am 19. August 1948)
}

1. In Abschnitt 4 ist in der Rechnung für reellen Zustand ein Fehler unterlaufen. In den beiden letzten Formeln auf S. 141 links unten muß es heißen: $1 / \cos ^{2} K^{\prime} a$ statt $-1 / \sin ^{2} K^{\prime} a$. Rechnet man hiermit richtig weiter, so fällt in (23) und (23a)

1 Z. Naturforschg. 3a, 134 [1948]. für den Streuquerschnitt $\sigma_{r}$ des reellen Zustandes im Korrekturglied des Zählers der Faktor 2 fort. Die B ethe sche Formel hierfür ist also in dieser Nöherung richtig.

In nächster Näherung erhält man, wenn man $\boldsymbol{k} a$ als von kleinerer oder glèicher Größenordnung wie 